\title{
FÓRUM
}

Submetido em 27.11.2013. Aprovado em 2.6.2014

Avaliado pelo sistema double blind review. Editores Científicos: Maria Tereza Saraiva de Souza, Orlando Cattini Junior e

José Carlos Barbieri

http://dx.doi.org/10.1590/S0034-759020140504

\section{COMO GESTORES HOSPITALARES UTILIZAM INDICADORES DE DESEMPENHO?}

\author{
How do hospital managers use performance indicators? \\ ¿Cómo los gestores hospitalarios utilizan los indicadores de desempeño?
}

\begin{abstract}
RESUMO
O objetivo principal deste artigo foi analisar como gestores de serviços hospitalares utilizam indicadores de desempenho para realizar a gestão estratégica de recursos sob a ótica da gestão do conhecimento baseada em evidências. Realizou-se uma pesquisa qualitativa, na qual foram investigados um sistema de indicadores hospitalares, um hospital público e outro filantrópico. A análise dos dados indicou que: o sistema estudado é utilizado como dispositivo de comparativos setoriais; gestores do hospital público usam indicadores como fonte de gerenciamento de recursos escassos e gestores do hospital filantrópico usam indicadores como fonte de informações para a sustentabilidade econômica e assistencial. A discussão dos resultados indicou que: o compartilhamento de informações é essencial para a aplicação do sistema de indicadores; o uso de indicadores no hospital público privilegia o desenvolvimento do conhecimento e o uso de indicadores no hospital filantrópico tem ênfase na gestão da qualidade.
\end{abstract}

PALAVRAS-CHAVE / Administração hospitalar, indicadores hospitalares, avaliação de desempenho hospitalar, gestão do conhecimento, gestão baseada em evidências.

\begin{abstract}
The main objective of this paper was to analyze how managers of hospital services use performance indicators to conduct the strategic management of resources by means of the perspective of evidence-based knowledge management. A qualitative study was performed to investigate a system of hospital indicators, a public hospital and a philanthropic hospital. The analysis showed that: the studied system is used as a comparative device sector, managers of the public hospital use indicators as a source of management of scarce resources and philanthropic hospital managers use indicators as a source of information for economic sustainability and caregiving. The discussion of results indicated that: information sharing is essential to the application of indicators system, the use of indicators in the public hospital privileges the development of knowledge and the use of indicators in the philanthropic hospital has an emphasis on quality management.
\end{abstract}

KEYWORDS I Hospital administration, hospital indicators, hospital performance evaluation, knowledge management, evidence-based management.

\section{RESUMEN}

El objetivo principal de este artículo fue analizar cómo los gestores de servicios hospitalarios utilizan los indicadores de desempeño para realizar la gestión estratégica de recursos bajo la óptica de la gestión del conocimiento basada en evidencias. Se realizó una encuesta cualitativa, en la cual se investigó a un sistema de indicadores hospitalarios, un hospital público y otro filantrópico. El análisis de los datos indicó que: el sistema estudiado es utilizado como dispositivo de comparativos sectoriales; gestores del hospital público usan indicadores como fuente de gestión de recursos escasos y gestores del hospital filantrópico usan indicadores como fuente de informaciones para la sostenibilidad económica y asistencial. La discusión de los resultados indicó que: compartir informaciones es esencial para la aplicación del sistema de indicadores; el uso de indicadores en el hospital público privilegia el desarrollo del conocimiento y el uso de indicadores en el hospital filantrópico tiene énfasis en la gestión de la calidad.

PALABRAS CLAVE I Administración hospitalaria, indicadores hospitalarios, evaluación de desempeño hospitalario, gestión del conocimiento, gestión basada en evidencias. 


\section{INTRODUÇÃO}

No Brasil, a demanda sobre serviços de saúde deve ser atendida segundo critérios de universalidade, integralidade e equidade (Lei n. 8.080, 1990), porém o sistema de saúde brasileiro sofre com problemas críticos e reincidentes, tais como: concentração de serviços em áreas urbanas; dimensionamento inadequado; sobreposição de ações e competição entre hospitais e a rede básica; definição de demandas influenciada pelo financiamento; insolvência financeira; baixa capacidade gerencial; resolutividade limitada e não observância do perfil epidemiológico regional (Ministério da Saúde, 2004). Esse quadro exige desafios aos gestores na busca de eficiência e eficácia na disponibilização e tratamento de informações para a melhoria da gestão hospitalar, seja no setor público ou privado (Cherchiglia \& Dallari, 2006; Cunha \& Corrêa, 2013).

A criação e a padronização de indicadores podem auxiliar na interação entre especialistas, pesquisadores e tomadores de decisão, possibilitando a geração de uma visão integrada que inove a elaboração do planejamento de serviços de saúde no Brasil (Báscolo, Yavich, \& León, 2006; Silva, Bezerra, Souza, \& Gonçalves, 2010). A complexidade dos sistemas de saúde, a escassez de recursos e a carência de gestão eficiente e eficaz justificam investimentos em análises de evidências que indiquem novos conhecimentos para agregar valor à saúde da população, ao setor e à sociedade (Corrao, Arcoraci, Arnone, Calvo, Scaglione, Bernardo, Legalla, Caputi \& Licata, 2009; Gonçalo \& Borges, 2010; Porter \& Teisberg, 2005; White, Lemak, \& Griffith, 2011).

Hospitais são serviços intensivos em conhecimento (Miles, Kastrinos, Flanagan, Bilderbeek, Hertog, Huntink \& Bouman, 1995). Essas organizações dependem do uso de conhecimento de alta complexidade; de informações que integrem as ações; da criação de conhecimento e da combinação entre conhecimentos dos especialistas, do setor, clientes e fornecedores (Gonçalo \& Borges, 2010; Milles et al., 1995; Porter \& Teisberg, 2005; Strambach, 2008). Em hospitais, a entrega é expertise, habilidade em pesquisa e desenvolvimento ou capacidade de resolução de problemas. 0 aprendizado pelo envolvimento entre especialistas e clientes ao longo do processo de entrega de serviços é fator crucial nessas organizações (Sveiby, 2008).

O objetivo principal deste estudo é analisar como gestores de serviços hospitalares utilizam indicadores de desempenho para realizar a administração estratégica de recursos sob a ótica da gestão do conhecimento baseada em evidências. Para atingi-lo, verificaram-se associações entre dimensões teóricas de Avaliação de Desempenho (AD), Administração Estratégica (AE) e Gestão do Conhecimento baseada em Evidências (GCE). Também, buscou-se compreender como essa dinâmica ocorre na prática gerencial de um grupo de hospitais que adotam um sistema de indicadores e em dois hospitais estudados em profundidade, um público e outro privado e filantrópico.

\section{REFERENCIAL TEÓRICO}

A capacidade de os hospitais reconhecerem suas características por meio de indicadores pode auxiliar na aquisição de desempenhos superiores, na criação de conhecimentos e na geração de evidências para orientar ações estratégicas. Neste item, é desenvolvido o referencial teórico conforme os eixos: AD hospitalar por meio de indicadores, AE de serviços hospitalares e GCE em serviços hospitalares.

\section{AD hospitalar por meio de indicadores}

Indicadores são medidas de desempenho que expressam resultados em índices numéricos (Hronec \& Andersen, 1994; Paladini, 2011). As práticas de AD hospitalar por meio de indicadores estão associadas a diversas abordagens: melhoria da qualidade em saúde, acreditação e certificação hospitalar (Forgia \& Couttolenc, 2009). Práticas de avaliação dessa natureza são conciliadas na abordagem da Total Quality Management (TQM). Esta se destaca por abranger ferramentas de gestão baseadas em indicadores focando a avaliação de processos, a partir de conhecimentos e práticas essenciais à organização e aos clientes (Forgia \& Couttolenc, 2009).

Juran e Gryna (1991) definiram a TQM como um prolongamento do planejamento dos negócios da empresa que inclui o planejamento da qualidade. São atividades da TQM: definição de objetivos, criação de estrutura gerencial, formação de pessoas e seleção de mecanismos de avaliação, entre os quais se destacam os indicadores de desempenho (Ahire, Golhar, \& Waller, 1996; Robin, 2008).

Para atingir a complexidade estratégica, é necessário aplicar diversos indicadores como instrumentos de gestão (Slack, Chambers, Harrison, \& Jhonston, 1997). 0 resultado de uma aplicação eficaz de indicadores é o aumento do conhecimento sobre pontos críticos nos processos, permitindo uma avaliação contínua da eficiência destes (Prahinsky \& Benton, 2004). Indicadores são, portanto, essenciais para proporcionar informações mensuráveis que permitam descrever a realidade organizacional.

Donabedian (1981) alertou sobre a necessidade de mensuração padronizada de indicadores para melhoria da qualidade em serviços de saúde. Passados 24 anos de pesquisas, Donabedian (2005) defendeu a avaliação multidimensional dos cuidados médicos com dados sobre estrutura, processos e resulta- 
dos. O uso de indicadores em sistemas integrados de informação pode melhorar o resultado financeiro dos hospitais e promover a transparência nas ações, caso sejam aliados à política institucional (Bittar, 2004; Wurster, Lichtenstein, \& Hogeboom, 2009). 0 monitoramento por indicadores pode contribuir para a melhoria da qualidade, produtividade e baixo custo nos serviços de saúde, quando combinado com outras ferramentas de gestão e alinhado à estratégia organizacional (Schout \& Novaes, 2007). Para tanto, é necessário: ter clara a perspectiva do significado que o indicador pretende refletir, definir quais aspectos do cuidado em saúde serão medidos e verificar quais são as evidências científicas disponíveis (Escrivão, 2007). Indicadores podem variar conforme as necessidades da organização, estando em consonância com as características e demandas de saúde da população.

Em hospitais, os fatores para o sucesso da TQM são: estrutura organizacional para identificar oportunidades de melhoria, estudos estatísticos e empowerment de equipes (Carman, Shortell, Foster, Hughes, Boerstler, O’Brien \& O’Connor, 2010). O envolvimento dos recursos humanos e da alta direção é crucial para a melhoria da qualidade assistencial e financeira.

A satisfação dos consumidores é o principal indicador de qualidade para fornecedores de saúde (Khattab \& Aborumman, 2011). Avaliação da qualidade em serviços de sáude implica a análise das intervenções dos especialistas em relação aos resultados percebidos pelos pacientes. A abordagem do Institute of Medicine [IOM] (2001) considera seis critérios baseados nos pacientes para a avaliação da qualidade em saúde: segurança, efetividade, cuidados, adequação no tempo, eficiência e equidade. Esses critérios são objetivos de qualidade em saúde pública.

Assim como médicos aplicam o conhecimento sobre anatomia humana para prevenir e restabelecer a saúde dos clientes, gestores podem usar o conhecimento sobre a "anatomia do desempenho" para prevenir problemas organizacionais e executar a melhoria da qualidade (Rummler \& Brache, 1992). Os programas de qualidade restringem-se, entretanto, ao diagnóstico e posicionamento organizacional baseados em modelos (Rummler \& Brache, 1992). Essa é apenas a primeira etapa da busca da qualidade nesses serviços e exige uma abordagem complexa com elementos para a melhoria efetiva.

0 modelo de gestão pela qualidade está ligado à evolução do uso, acesso e transferência da informação veiculada nos sistemas de indicadores (Gonçalo \& Borges, 2010). Esses autores consideram organizações de saúde com ênfase em informação como organizações tradicionais. A complexidade dos serviços hospitalares demanda que sejam concebidos como organizações de aprendizagem (Gonçalo \& Borges, 2010). Tais organizações são organizações intensivas em conhecimento. O uso de ferramentas de compartilhamento do conhecimento pode contribuir com a redução das diferenças culturais, econômicas e sociais entre profissionais e usuários dos serviços de saúde (Gurgel \& Vieira, 2002).

\section{AE de serviços hospitalares}

Na visão baseada em recursos (Barney, 1991; Grant, 1996), o conhecimento é recurso estratégico. Em serviços intensivos em conhecimento, como são os hospitais, a entrega de valor baseia-se nas estratégias de criação e compartilhamento do conhecimento. Estas ocorrem em contextos e dispositivos oferecidos pela organização (Nonaka \& Krogh, 2009; Nonaka \& Takeuchi, 1997).

Em um estudo sobre desempenho hospitalar brasileiro (Forgia \& Couttolenc, 2009), verificou-se que o controle de recursos é adquirido pela aquisição de habilidades, gestão do conhecimento, aprendizagem e know-how. 0 gerenciamento desses recursos e sua conversão em serviços poderão equilibrar a influência do ambiente na estrutura organizacional.

0 trabalho de Sveiby (2008) mostra resultados sobre o valor criado pela gestão do conhecimento com base em novas políticas e processos. 0 autor concluiu que, pelo compartilhamento de experiências entre pacientes com alta cirúrgica e novos pacientes e pela transferência de conhecimento entre médicos e enfermeiros, foi possível reduzir o temor de novos pacientes, diminuir custos com medicamentos e diluir a demanda sobre os profissionais. Essas evidências indicam redução do tempo do ciclo de serviços, redução de custos, aumento no retorno de investimentos, aumento da satisfação dos clientes e geração de serviços de excelência.

Porter e Teisberg (2005), ao considerarem a cadeia de valor como ferramenta primordial para delinear e analisar os processos de entrega em saúde, incluem o desenvolvimento do conhecimento como suporte para melhorar resultados. São atividades como: medidas e rastreamento, treinamento do pessoal, desenvolvimento de tecnologia e melhoria de processos. A cadeia de valor deve iniciar com monitoramento e prevenção. Entre esses estágios, há três tipos de atividades: acessibilidade (consultas presenciais, transportes, visitas domiciliares, monitoramento e consultas via internet, por exemplo), medição (das circunstâncias médicas do paciente) e informação (atividades de notificação, educação e instruções ao paciente). Essa lógica de competitividade (Porter \& Teisberg, 2005) implica ações integradas entre fornecedores para a melhoria conjunta do setor e da saúde da população.

\section{GCE em serviços hospitalares}

Aprender com o erro e reorientar ações ampliando conhecimentos para a aquisição de melhorias é fator crítico em estudos so- 
bre gestão por evidências (Corrao et al., 2009; Pfeffer \& Sutton, 2006; Reay, Berta, \& Kohn, 2009; Rousseau, Manning, \& Denyer, 2008; White, Lemak, \& Griffith, 2011). As decisões em serviços médicos devem ser baseadas no conhecimento atualizado e comprovado como o mais resolutivo (Corrao et al., 2009).

Hospitais podem usar a medicina baseada em evidências para superar barreiras, gerando treinamento e tecnologias para que equipes atinjam melhores resultados. A colaboração, a renovação e a transferência de conhecimentos entre especialistas e gestores para a aplicação de evidências, também, podem contribuir com o gerenciamento dessas organizações, levando-as ao encontro da entrega de qualidade (Kovner, Pfeffer, \& Fine, 2010; Pfeffer \& Sutton, 2006).

0 conhecimento é crucial na tomada de decisão em serviços de saúde e é a raiz da medicina baseada em evidências (Corrao et al., 2009). 0 estudo de Corrao e outros (2009) propõe uma abordagem pragmática para a gestão de serviços hospitalares por intermédio de um novo paradigma: Gestão do Conhecimento baseada em Evidências (GCE). Evidências adquirem o status de informações transformadas em conhecimento.

Na GCE em serviços de saúde (Corrao et al., 2009), o foco é a ampliação do know-how para a melhoria dos sistemas, organizações e gestão de recursos econômicos e humanos. Na prática, requer a criação de uma estrutura de governança estratégica ancorada em um sistema capaz de coletar e tratar dados, prover informações e conhecimentos. Essa estrutura compreende os seguintes elementos: definir tarefas, auditoria, eficiência clínica, eficácia, pesquisa e desenvolvimento e gestão de riscos (Corrao et al., 2009). A governança deve permear os seguintes pilares: GCE, gestão da qualidade e consultoria e formação médica continuada.

A estrutura só será eficaz se houver uma cultura de governança baseada em evidências desde os níveis operacionais, passando pelos sistemas de apoio ao diagnóstico, administrativo e sustentada pela alta gestão (Corrao et al., 2009). Essa abordagem promove melhores desempenhos organizacionais por meio da mudança na cultura organizacional e de métodos de gestão. Sistemas de educação continuada são centrais para a cultura de gestão por evidências, com modificação na gestão administrativa e clínica, desenvolvimento de lideranças e responsabilidade gerencial compartilhada, monitoramento financeiro, dimensionamento adequado da demanda, prestação eficiente e eficaz dos serviços com controle por protocolos, auditoria, integração estratégica e manutenção da melhoria (White, Lemak, \& Griffith, 2011).

Reay et al., (2009) pesquisaram temas sobre a influência da gestão por evidências no desempenho organizacional. No estudo, os autores encontraram investigações sobre como organizações tomam decisões com o uso de novas tecnologias, uso de sistemáticas para gerir empresas, influências de evidências em tomada de decisões em recursos humanos, finanças, controladoria e gestão de saúde e sobre tradução do conhecimento e aprendizagem como forma de aquisição de melhorias.

O detalhamento sobre como gestores hospitalares utilizam indicadores de desempenho para realizar a gestão estratégica de recursos não é explicitado pelos estudos citados no presente referencial teórico. Tal investigação é objeto deste artigo.

\section{Síntese do referencial teórico}

O Quadro 1 exibe o resumo do referencial teórico com os devidos eixos, os principais conceitos e suas principais contribuições para a gestão de serviços hospitalares.

O foco na TQM é associado à avaliação de desempenho, enfatizando a informação adquirida por meio de indicadores em organizações tradicionais (Gonçalo \& Borges, 2010; Juran \& Gryna, 1991). Estratégia é a orientação dos recursos organizacionais avaliada criticamente a partir dos resultados dos indicadores, conhecimentos e evidências orientadoras das ações organizacionais no ambiente interno e em relação ao meio externo (Barney, 1991; Grant, 1996). Conhecimento é o elemento central para a gestão de organizações intensivas em conhecimento (Miles, et al., 1995). Evidências representam o conhecimento comprovado como resolutivo. A linha pontilhada que divide os temas "gestão do conhecimento" e "baseada em evidências" indica que ambos são complementares e compõem um novo paradigma (Corrao et al., 2009). Serviços hospitalares promovem o exercício prático da estratégia organizacional por meio de aquisição de conhecimentos, know-how e aprendizagem (Gonçalo \& Borges, 2010).

\section{PROCEDIMENTOS METODOLÓGICOS}

Nesta seção, apresenta-se a classificação da pesquisa, são descritas as três etapas da investigação e explicitados os procedimentos metodológicos. Optou-se pela divisão em etapas por conta das diferentes modalidades de investigação demandadas pela complexidade da realidade investigada. Reuniões periódicas com o comitê gestor do sistema de indicadores e as diretorias dos hospitais estudados em profundidade foram realizadas para viabilizar o estudo. Além disso, nas Etapas 2 e 3, as organizações envolvidas exigiram um processo de aprovação pelos respectivos comitês de ética em pesquisa e ocorreram visitas sistemáticas para a realização das entrevistas e observações. 
Quadro 1. Síntese do referencial teórico

\begin{tabular}{|c|c|c|}
\hline EIXO TEÓRICO & PRINCIPAIS CONCEITOS & PRINCIPAIS CONTRIBUIÇÕES \\
\hline $\begin{array}{l}\text { Avaliação de } \\
\text { Desempenho hospitalar } \\
\text { por meio de indicadores } \\
\text { (AD) }\end{array}$ & Informação & $\begin{array}{l}\text { Foco na gestão da qualidade e na } T Q M \text {, avaliação de custos, flexibilidade, preço; } \\
\text { avaliação, controle e planejamento; identificação de pontos críticos; envolvimento } \\
\text { dos recursos humanos para promover melhoria contínua, eficiência e eficácia de } \\
\text { processos. }\end{array}$ \\
\hline $\begin{array}{l}\text { Administração } \\
\text { Estratégica de serviços } \\
\text { hospitalares } \\
\text { (AE) }\end{array}$ & Estratégia & $\begin{array}{l}\text { Conhecimento como recurso estratégico. } \\
\text { Desenvolvimento do conhecimento é crucial para agregar valor em serviços } \\
\text { hospitalares. } \\
\text { Controle de recursos escassos deve ser acessado pela aquisição de habilidades, } \\
\text { criação e compartilhamento do conhecimento, aprendizagem e know-how. }\end{array}$ \\
\hline Gestão do conhecimento & Conhecimento & $\begin{array}{l}\text { Foco em serviços intensivos em conhecimento, nos processos de criação do } \\
\text { conhecimento, na aprendizagem organizacional e no compartilhamento do } \\
\text { conhecimento entre especialistas e clientes como fonte de sustentabilidade } \\
\text { assistencial, financeira e excelência na prestação de serviços. }\end{array}$ \\
\hline
\end{tabular}

\section{Classificação da pesquisa}

Quanto à finalidade, o presente estudo é uma pesquisa aplicada, pois é motivado pela compreensão de um problema prático (Miguel, 2012; Zanella, 2009). Para atingir o objetivo, a investigação ocorre na prática dos gestores hospitalares, visando compreender o uso de indicadores no setor hospitalar.

Quanto ao método, a pesquisa é qualitativa (Miguel, 2012), pois visa explicar como gestores hospitalares utilizam indicadores de desempenho. Esse tipo de análise é baseado em conhecimentos teóricos e empíricos para gerar cientificidade (Richardson, 2007; Zanella, 2009). Sua preocupação básica é o mundo empírico e o seu ambiente natural (Zanella, 2009), neste caso, o ambiente hospitalar. Neste estudo, portanto, a pesquisa qualitativa consiste em um agrupamento de práticas materiais (entrevistas) e interpretativas (análise de conteúdo) para estudar o fenômeno do uso de indicadores de desempenho e seus significados (Denzin \& Lincoln, 2007).

Quanto à estratégia, esta pesquisa é exploratória (Zanella, 2009), pois pretende-se ampliar o conhecimento sobre 0 tema estudado. 0 estudo ocorre por meio da exploração da realidade e se propõe a interpretá-la além da informação chegando ao conhecimento experienciado pelos sujeitos pesquisados.

Quanto aos procedimentos de coleta de dados houve pesquisa bibliográfica, pesquisa documental e pesquisa de campo (Flick, 2014). Foram consultadas publicações sobre os eixos teóricos do estudo, registros históricos dos hospitais nos respectivos sites e documentos para descrever as instituições pesquisadas, e realizaram-se entrevistas com gestores hospitalares. 0 Quadro 2 mostra a classificação da pesquisa:

\section{Quadro 2. Classificação da pesquisa}

\begin{tabular}{l|l}
\hline CRITÉRIO & CLASSIFICAÇÃo \\
\hline Finalidade & Aplicada \\
\hline Método & Qualitativa \\
\hline Estratégia & Exploratória \\
\hline Coleta de dados & Pesquisa bibliográfica, documental e de campo \\
\hline
\end{tabular}

\section{Etapa 1: estudo exploratório em nove hospitais que usam um sistema de indicadores}

Consistiu na investigação sobre uso gerencial de indicadores de desempenho hospitalar por meio de entrevistas em profundidade com gestores de nove hospitais brasileiros credenciados no Sistema de Indicadores Padronizados para a Gestão Hospitalar [SIPAGEH] (2008). Os hospitais foram contatados previamente de modo aleatório e foi possível investigar, por adesão à pesquisa, nessa etapa, nove hospitais, dos 41 que pertencem ao SIPAGEH. Destes, sete são entidades beneficentes sem fins lucrativos, um é uma fundação universitária e um, uma entidade privada sem fins lucrativos. Foram entrevistados gestores dos níveis de superintendência (um), diretoria (cinco), gerência de controladoria (um), responsáveis pelo departamento de tecnologia da informação (dois), totalizando nove entrevistas. Os hospitais investiga- 
dos estão nas regiões Sul, Centro e Sudeste do País. Nessa etapa, foi aplicada a seguinte pergunta: Como você utiliza os indicadores de desempenho do SIPAGEH na gestão hospitalar?

\section{Consolidação das dimensões teóricas, categorias de pesquisa e definição do instrumento de coleta de dados}

A Etapa 1, além de proporcionar a exploração sobre a utilização de um sistema de indicadores de desempenho hospitalar a partir da experiência prática dos gestores, possibilitou, com o apoio do software NVIVO9 ${ }^{\circledR}$, a consolidação das dimensões teóricas, das respectivas variáveis de pesquisa e a definição de um roteiro de entrevistas para aprofundar a investigação em dois hospitais gerais, um público e outro privado sem fins lucrativos (Etapas 2 e 3 ). Elaborou-se um roteiro de entrevistas em profundidade composto por 13 perguntas, conforme exibe o Quadro 3.

\section{Seleção dos casos para as Etapas 2 e 3}

Para tais etapas, foi utilizado o método de estudo de casos (Yin, 2001). A escolha das organizações a serem estudadas deu-se por seu contexto ser representativo de hospitais gerais públicos, filantrópicos e privados, sendo estas as configurações administrativas da maioria dos hospitais brasi- leiros (Forgia \& Couttolenc, 2009). Os hospitais escolhidos possuem sistemas de gestão com apoio de indicadores e know-how quanto ao uso destes, sendo reveladores (Miguel, 2012) do objetivo deste artigo. As Etapas 2 e 3 foram concomitantes, entretanto optou-se por discriminá-las, devido às diferenças nas características dos modelos de gestão dos hospitais.

\section{Etapa 2: estudo de caso em um hospital geral público}

Consistiu na investigação sobre uso de indicadores de desempenho hospitalar em um hospital geral público brasileiro que atende o Sistema Único de Saúde (SUS). Adota o modelo de gestão participativa e inclui avaliação de qualidade, produtividade e assistência, avaliação do desenvolvimento individual, das equipes e dos gestores por meio de indicadores e formação continuada. No modelo de gestão, são previstos colegiados com vistas à tomada de decisões coletivas e democráticas.

Foram entrevistados 10 gerentes e líderes dos trabalhadores nos colegiados de gestão, conforme as divisões de Unidades de Pacientes Externos (Emergência e Ambulatório), Unidades de Internação, Serviços de Apoio a Diagnóstico e Tratamento e Ensino e Pesquisa.

\section{Quadro 3. Roteiro de entrevistas em profundidade}

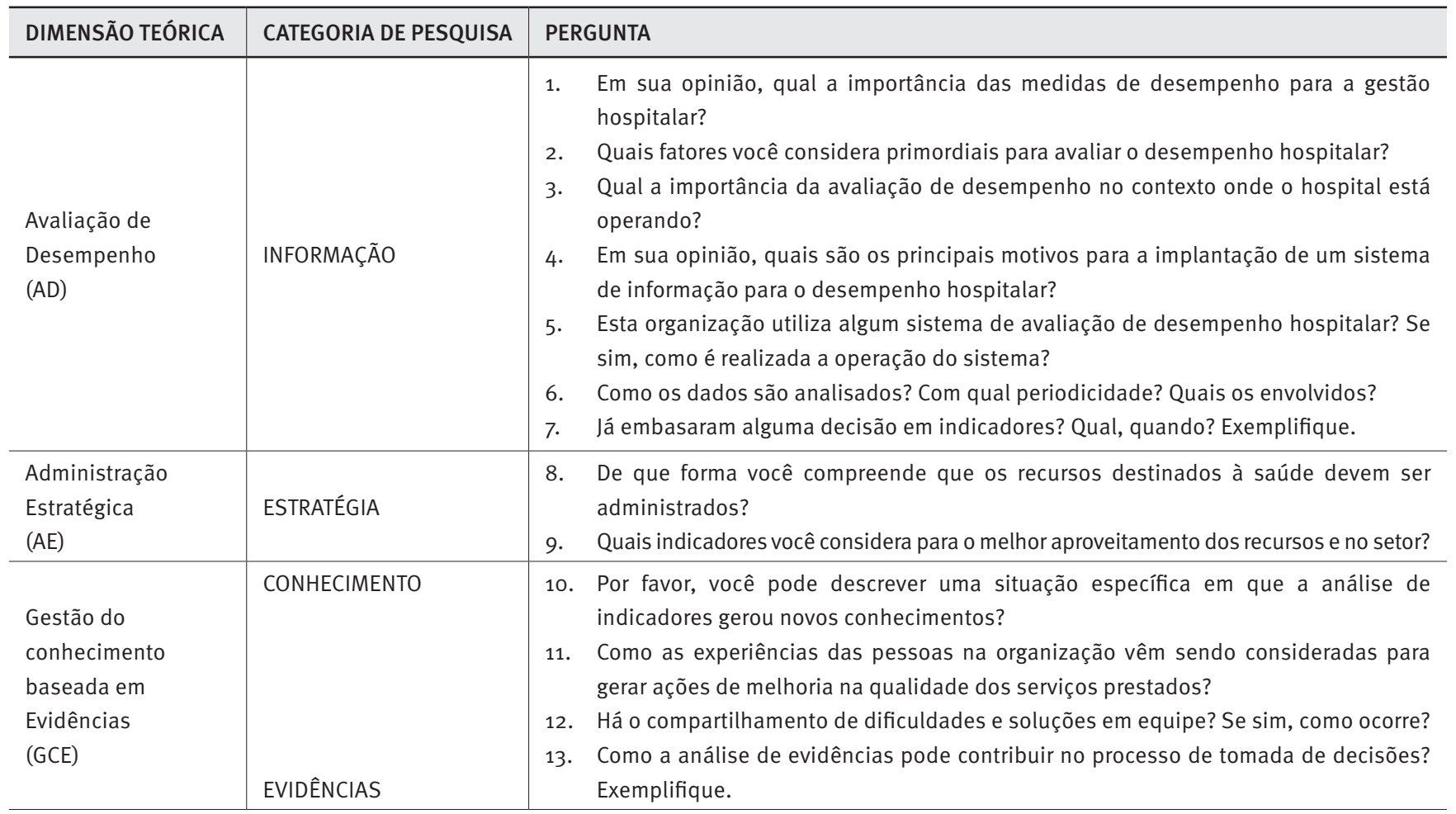




\section{Etapa 3: estudo de caso em um hospital geral filantrópico e privado}

Consistiu na investigação sobre o uso de indicadores de desempenho hospitalar em um hospital geral filantrópico brasileiro. O hospital destina 60\% de suas instalações ao SUS e os outros $40 \%$ para pacientes de convênios e particulares, por conta da legislação de filantropia em saúde. Adota o modelo de gestão corporativa, sendo dividido em unidades de negócios monitoradas por indicadores da qualidade, assistencial, de recursos humanos e financeiros. Possui plano estratégico para melhorar continuamente a saúde da população por intermédio da cultura da excelência, de ações de promoção da saúde, fidelização de clientes e busca por melhores resultados econômicos.

Foram entrevistados oito gestores e lideranças das seguintes áreas: Direção (Direção Médica, Ensino e Pesquisa), Administração, Bloco Cirúrgico, Unidade de Internação e Convênios, Unidade Materno-Infantil, Emergência e Serviço Social.

\section{Tratamento dos dados}

As entrevistas foram gravadas, transcritas e inseridas no software NVIVO9 ${ }^{\circledR}$. Após a realização da Etapa 1 , com os resultados parciais, a definição das dimensões teóricas, das respectivas categorias e da consolidação do roteiro de entrevistas, o software foi utilizado para a categorização e análise das entrevistas. Selecionaram-se termos por frequência no discurso dos entrevistados das Etapas 2 e 3, respectivamente, para gerar redes de associações entre as dimensões teóricas contendo as respectivas variáveis. Estabeleceram-se associações implícitas entre as dimensões (AD, AE e GCE) no cotidiano de trabalho dos gestores dos dois hospitais estudados em profundidade e a interpretação do conteúdo das entrevistas (Bardin, 2011).

A Figura 1 mostra a dinâmica da estratégia de pesquisa:

Figura 1. Estratégia de pesquisa

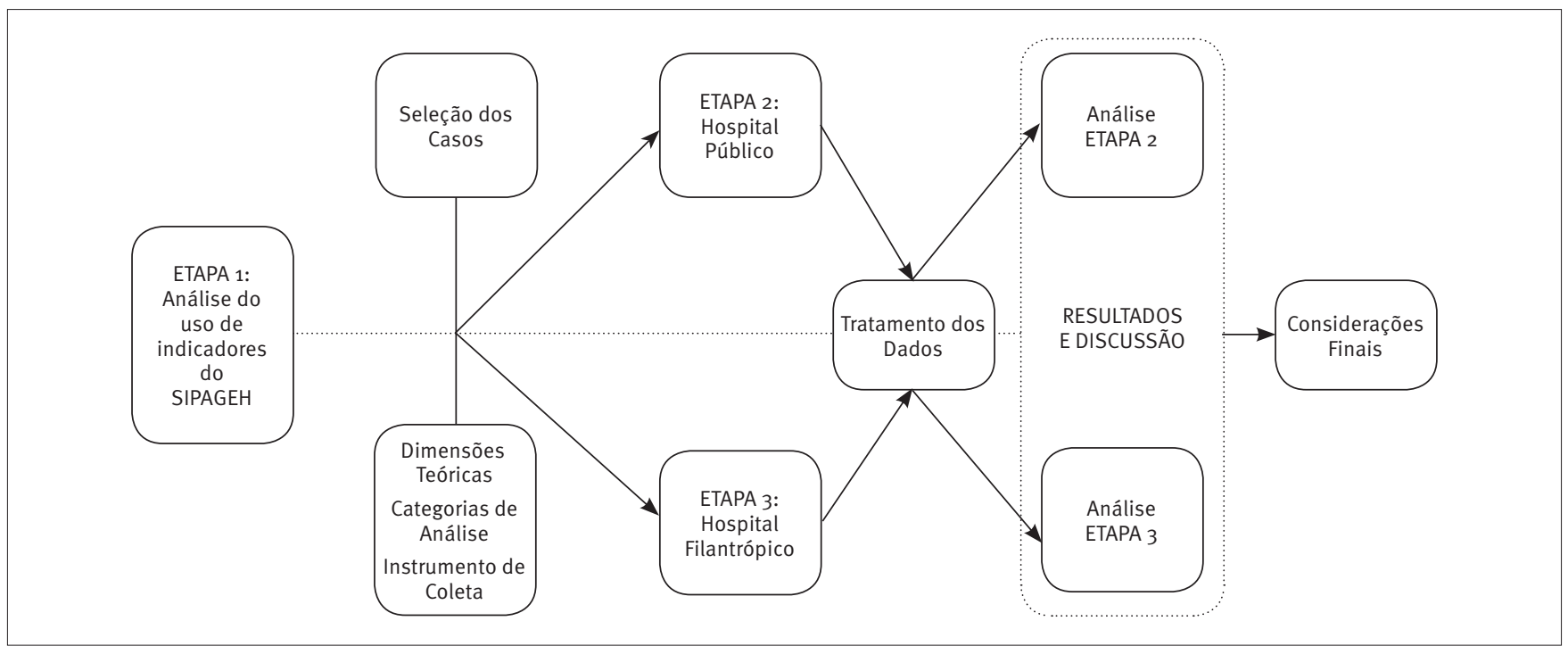

\section{APRESENTAÇÃO E DISCUSSÃO DOS RESULTADOS}

Nesta seção, apresentam-se e discutem-se os resultados da pesquisa dentro das respectivas etapas. Para a Etapa 1, foi analisado o conteúdo das entrevistas, definido o roteiro de entrevistas e consolidadas as dimensões e categorias de pesquisa. Nas Etapas 2 e 3, com o aprofundamento da pesquisa, acrescentou-se a análise das redes de associações entre as dimensões teóricas do estudo identificadas nas entrevistas e selecionaram-se os principais indicadores utilizados pelos gestores dos respectivos hospitais segundo o contexto estratégico onde estão inseridos.

\section{Etapa 1}

Quanto ao conteúdo, pode-se interpretar que, em primeiro lugar, as organizações investigadas utilizam SIPAGEH para avaliar desempenho: “[...] tendo acesso a indicadores, nós podemos ter um comparativo para avaliar o desempenho do hospital perante os outros, que serve como estímulo para conquistar meIhores resultados". Nessa fala de um entrevistado, observa-se 
que indicadores do SIPAGEH são utilizados para realização de benchmarking, ou seja, estudos comparativos visando à melhoria de resultados.

Em segundo lugar, o sistema é utilizado na aquisição de conhecimentos para orientar decisões baseadas em evidências: "[...] o sistema auxiliou, pois começamos a estudar estatísticas, nomenclaturas, maneiras de calcular. Induziu as pessoas a aprenderem mais, porque tem que saber analisar uma alta, uma diária, uma internação. Ele trouxe esse conhecimento de saber exatamente com o que estás trabalhando, do gestor pegar o dado e saber decidir". Observa-se, nessa fala, o intuito de gerar e gerir o conhecimento a fim de agir estrategicamente na tomada de decisões.

Prevalece, entretanto, a coleta de informações, e uma evolução para a aplicação da GCE depende de critérios como: ampliação de pesquisas sobre dados inseridos no SIPAGEH, ampliação do compartilhamento de informações entre participantes com resguardo de sigilo e investimento em formação aos profissionais da saúde para uso do sistema e interpretação de resultados dos indicadores: “[...] compartilhamos mais na instituição, com a diretoria e com o grupo de colaboradores. Com outras instituições é bem pouco"; "Quando se trata de indicadores gerenciais, há desconfiança, temor de que o sigilo sobre a estratégia seja rompido"; “[...] na formação dos profis- sionais de saúde fica uma resistência, faz treinamento e continuam não sabendo".

A mudança cultural (Corrao et al., 2009) é mencionada como determinante e favorável à análise de evidências para melhoria da qualidade e resultados estratégicos no setor hospitalar: "É difícil os hospitais mostrarem suas dificuldades. Precisaria de um parâmetro para um hospital em relação a pagamento, contrato de manutenção de equipamentos... No futuro vai ser melhorado, porque a concorrência é pouca perto da demanda".

Observa-se que a competição baseada em valor por meio de ações conjuntas e integradas entre fornecedores de saúde (Porter \& Teisberg, 2005) é um elemento a ser exercido com maior intensidade entre os participantes do SIPAGEH. Os entrevistados dessa etapa utilizam indicadores como dispositivos para comparativos setoriais, sem o aprofundamento dos resultados para criar e compartilhar conhecimentos e evidências.

\section{Etapa 2}

O estudo dessa etapa mostrou associações entre os termos das entrevistas e as devidas dimensões teóricas, conforme ilustra a Figura 2:

\section{Figura 2. Rede de associações - etapa 2}

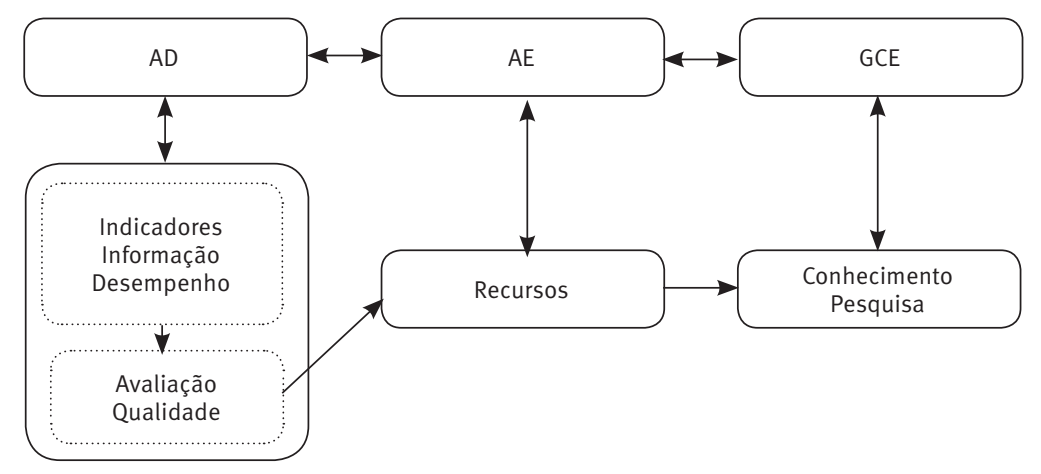

Interpretando a Figura 2, verticalmente, estão os termos associados ao tema da pesquisa e, horizontalmente, as dimensões teóricas com as respectivas redes associativas.

$\mathrm{Na}$ Figura 2, a dimensão AD exibe associação do termo “indicadores” com “informação” e este, por sua vez, é ligado ao "desempenho". "Avaliação" e "qualidade” são associados aos "recursos", portanto fazem ligação da dimensão AD com
$A E$, a qual está conectada com GCE, representada por "conhecimento" e "pesquisa”. Assim, nesse caso, avaliação da qualidade dos recursos organizacionais é complementada e fomentada por conhecimento e pesquisa.

Esses achados corroboram as afirmações teóricas sobre a importância do uso dos sistemas de informação baseados em indicadores e do conhecimento sobre desempenho para 
prevenir problemas e realizar melhorias organizacionais e setoriais (Donabedian, 2005; Rummler \& Brache, 1992). 0 controle de recursos escassos é acessado pela aquisição de habilidades, gestão do conhecimento, know-how e aprendizagem, como no exemplo a seguir:

É muito difícil, pois todo o recurso é apertado. Tem a questão de distribuição de recursos humanos nos serviços. Levantamos as necessidades. A discussão é árdua entre os gerentes. Que indicador vamos usar? Vamos ver onde há áreas de carência e onde vou colocar recursos para sanar isto. 0 critério pode ser absoluta carência, deficiência, desqualificação ou serviços que integram necessidades de várias gerências. Reestruturar alguns serviços, às vezes, também ajuda.

Segundo os entrevistados, evidências oriundas de métodos de mensuração estatística são limitadas para orientar ações organizacionais em diferentes contextos. 0 diálogo a seguir ilustra esse comentário: "P: Há evidências para dizer que o fato de as equipes estarem acompanhadas sempre pelo médico garanta a horizontalidade e um melhor fluxo de troca de informações e soluções no hospital? R: Poderia. Só que há várias coisas subjetivas, do indivíduo, como empatia. Há várias coisas da organização, da hierarquia, das relações de poder, depois infraestrutura, momento da vida das pessoas, do clima da organização. Eu não posso isolar estas variáveis".

Salienta-se que, apesar da incompletude das informações e de evidências serem refutáveis, é importante implantar estudos pilotos e experimentos para a aprendizagem organizacional (Reay et al., 2009). A produção de evidências pode facilitar decisões, minimizando influências negativas do ambiente externo e da informalidade sobre organizações de saúde (Pfeffer \& Sutton, 2006).

Entende-se que a organização estudada busca, por meio da gestão participativa e do desenvolvimento de recursos humanos, melhorar continuamente a assistência em saúde, entretanto há barreiras quanto ao fluxo de informações (Nonaka e Krogh, 2009; Nonaka \& Takeuchi, 1997) entre os diferentes serviços do hospital e deste para com o restante da rede de saúde: "Os serviços têm suas atribuições, mas não está existindo um fluxo adequado de comunicação entre os diferentes setores. Isto é um ponto crítico que precisa melhorar para desenvolver um pouco mais de agilidade nas ações".

Há experiências baseadas em indicadores para dimensionar a demanda, qualificar o atendimento e alocar recursos junto ao sistema financiador, porém a cultura da gestão por evidências (Corrao et al., 2009) não é consolidada, devido aos aspectos subjetivos e contextuais. A política de desenvolvimento institucional carece da conversão das experiências individuais e coletivas em evidências: "Existe uma gestão compartilhada, mas não integrada e, às vezes, ela se integra na informalidade. Vamos fazer uma reunião, mas esquecemos de convidar um setor importante. Se fosse sistematizado, seria melhor. Precisamos procurar informação e conhecimento e estabelecer um canal" ; "Tentamos vários mecanismos para compartilhar as decisões, mas temos dificuldade"; "Acho que tem várias questões nesta coisa da produção do conhecimento da hierarquia do poder baseado em saber que impede que se pense o conhecimento para todos".

No contexto da saúde pública, estratégias de recursos humanos demandam que o compartilhamento de problemas e soluções supere resistências ideológicas e diferenças entre categorias profissionais (Gurgel \& Vieira, 2002; Pfeffer \& Sutton, 2006; Rousseau et al., 2008). Planos de desenvolvimento estão sendo criados e evoluem, apesar das dificuldades inerentes à gestão de pessoas, ao processo de mudança e à escassez de recursos na saúde brasileira.

De acordo com as experiências relatadas pelos entrevistados da Etapa 2, os indicadores apresentados no Quadro 4 foram considerados fundamentais à avaliação do desempenho no hospital.

Observa-se, no Quadro 4, que a aplicação dos indicadores sugeridos pelos entrevistados poderá contribuir no redimensionamento das capacidades, competências, recursos humanos, qualidade de atendimento, previsão de custos e aporte financeiro para a melhoria do desempenho dos serviços prestados. Para que essas práticas sejam estabelecidas, é necessário melhorar a adesão ao uso de indicadores para orientações estratégicas ao longo do tempo.

\section{Etapa 3}

Nessa etapa, verificaram-se associações entre termos destacados nas entrevistas e as devidas dimensões de teóricas, conforme a Figura 3.

$\mathrm{Na}$ Figura 3, observa-se que a dimensão $A D$ associa-se à AE ("estratégia") por meio dos termos "desempenho" e "qualidade". "Indicadores" é associado ao "conhecimento" e "evidências", indicando proximidade entre AD e GCE. "Informação" apresenta associação com "compartilhamento". Isso mostra que, embora "desempenho" e "qualidade" sejam cruciais para a estratégia organizacional, o apoio de indicadores gera "conhecimento" e "evidências". "Informações" compar- 
tilhadas completam o ciclo, denotando que AD e GCE estão a serviço da "estratégia".

Figura 3. Rede de associações - etapa 3

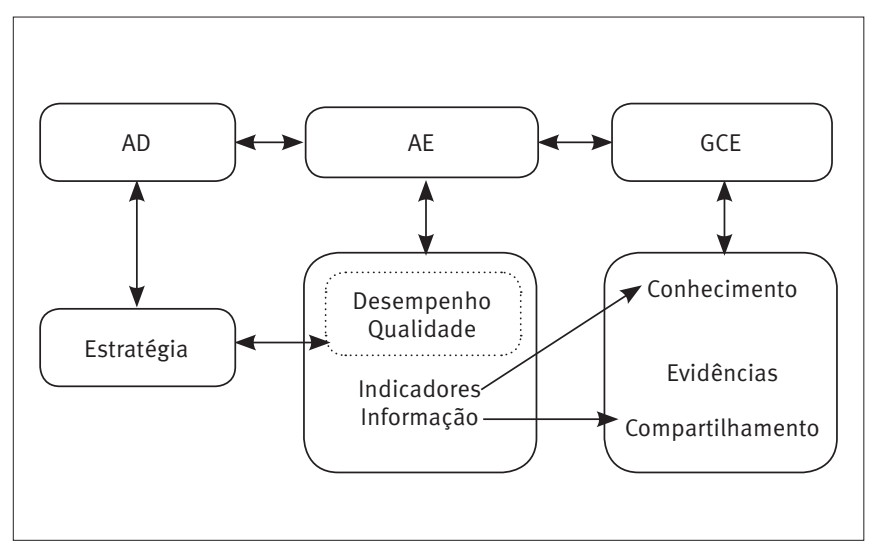

Um indicativo de melhorias nos critérios estratégicos (Carman et al., 2010; Khattab \& Aborumman, 2011) do hospital - qualidade assistencial, autossustentação e responsabilidade social - é identificado no processo de articulação entre as três dimensões. A gestão da qualidade (Ahire et al., 1996; Robin, 2008) é aplicada pela interpretação de resultados e melhoria contínua do desempenho visando à sustentabilidade:

[...] são indicadores de gestão, de desempenho econômico e da qualidade de assistência. Seria impossivel a organização sobreviver sem histórico de desempenho e ver aonde ela quer chegar. Tão importante quanto conhecer cenários do negócio da instituição, é conseguir analisar com isenção o seu desempenho para decidir o seu rumo.

O uso de indicadores para gerar conhecimentos, meIhoria da qualidade, aquisição de resultados assistenciais e econômicos positivos é fator crucial em hospitais, pela complexidade das operações e finalidade assistencial. A busca pelo compartilhamento de informações colhidas de indicadores assistenciais e econômicos visa a que o conhecimento seja criado para ser convertido em evidências que possam orientar a organização na captação de recursos. A organização procura conciliar a função assistencial como conveniada ao SUS com a sustentabilidade orientada pela visão de negócio.

Para superar dificuldades financeiras, os gestores utilizam, também, a tradição em ensino e pesquisa que o hos- pital possui no cenário da saúde nacional e internacional. 0 conhecimento armazenado foi decisivo para o comprometimento com as exigências do SUS e a garantia do atendimento qualificado: "Como demos origem a faculdades de medicina, o conhecimento que há aqui é muito grande. Esse conhecimento foi explorado para que as pessoas se comprometessem com a instituição no momento em que passamos a atender o SUS". O uso de evidências para orientar rumos (Kovner et al., 2010; Reay et al., 2009) não supera, porém, normas e protocolos médicos: “[...] evidências, resultados como algum problema, mudança em alguma unidade? Hoje será apresentada a cirurgia segura. Isso vira uma faixa institucional, todos os meses tem isso. Temos gerenciamento às diretrizes, gerenciamento à rotina".

Há que se conciliar a cultura imediatista da solução de problemas de saúde com planejamento estratégico e envolvimento das equipes (Gurgel \& Vieira, 2002) na criação de conhecimentos (Nonaka \& Krogh, 2009) que renovem pesquisas e experiências acumuladas ao longo da história do hospital: "O hospital deveria fazer mais reuniões para tomar decisões de áreas, campos de atuação, aprofundamento específico por área. Tem que ter conhecimento! A pesquisa é a base de dados que gera conhecimento. E este vai gerar possibilidade de tomada de decisão. Se tomar decisão sem base de dados ou se a base de dados for construída em cima de falta de pesquisa, ela vai ficar fora da realidade e as decisões serão tomadas de forma equivocada"; "Isso é falta de uma cultura! É uma correria! O profissional vai para outro hospital e fica menos interessado em gerar soluções".

Destacam-se a certificação nos critérios da TQM (Juran \& Gryna, 1991) e o monitoramento dos desempenhos operacional e financeiro. A iniciativa de compartilhar informações sobre desempenho e experiências com outras organizações do setor, também, é fator relevante para a criação de conhecimentos que garantam a qualidade e a sustentabilidade econômica.

O Quadro 5 mostra os indicadores mencionados pelos entrevistados na Etapa 3 como cruciais para orientar ações de assistência em saúde da população e para a sustentabilidade do hospital.

Observa-se, no Quadro 5, que os principais indicadores para a mensuração de resultados e melhoria da sustentabilidade são de qualidade da assistência e desempenho econômico, conforme nomeados pelos entrevistados. As ações estratégicas são orientadas pelos princípios da TQM, entretanto dificuldades para integrar a informação internamente parecem ser desafios aos esforços de cumprimento de metas para desempenhar o planejado. 
Quadro 4. Indicadores - etapa 2

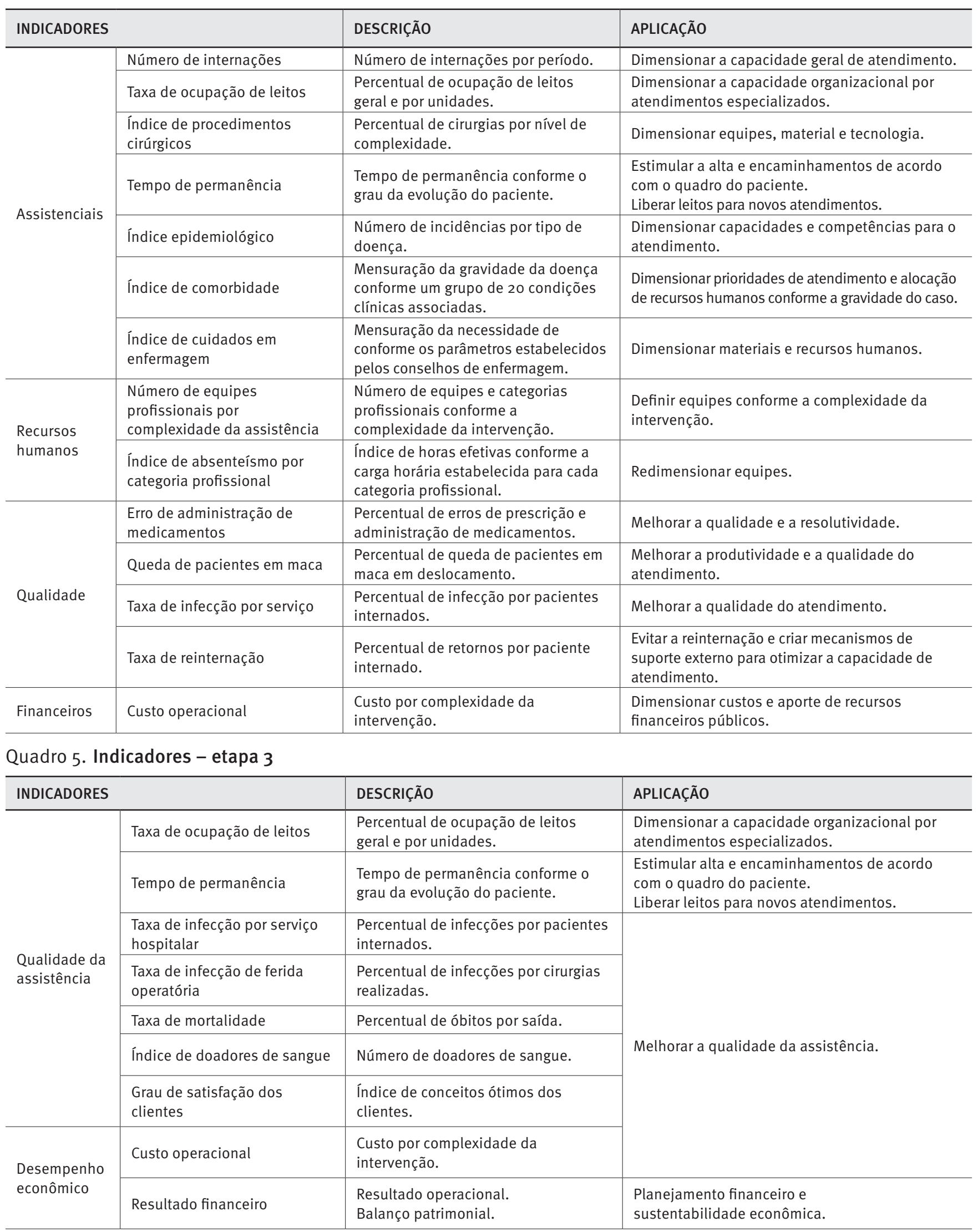




\section{CONSIDERAÇÕES FINAIS}

O objetivo do artigo foi analisar como gestores de serviços hospitalares utilizam indicadores de desempenho para realizar a administração estratégica de recursos sob a ótica da gestão do conhecimento baseada em evidências. 0 método consistiu em uma pesquisa exploratória, envolvendo três etapas: uma investigação inicial em um sistema de indicadores e dois estudos de caso em dois hospitais gerais, um público e outro filantrópico. Os dados de entrevistas em profundidade com gestores hospitalares foram tratados com o apoio do software para pesquisas qualitativas NVIVO ${ }^{\circledR}$ Estabeleceu-se como dimensões teóricas: Avaliação de Desempenho (AD), Administração Estratégica (AE) e Gestão do Conhecimento baseada em Evidências (GCE) para fundamentar o roteiro de entrevistas e a análise dos dados.

$\mathrm{Na}$ Etapa 1, consolidaram-se as categorias de pesquisa e definiu-se o roteiro de entrevistas. Além disso, ficou entendido que um sistema de indicadores é essencial para comparações estratégicas, a aplicação da GCE com apoio do SIPAGEH é incipiente, a aplicação dos resultados dos indicadores do sistema como fonte para conhecimentos resolutivos depende da ampliação da pesquisa e do compartilhamento de informações entre os participantes. Indicadores do SIPAGEH são utilizados como dispositivo para realizar comparativos, sem o aprofundamento dos resultados para criar e compartilhar conhecimentos e evidências. Os entrevistados apontam a necessidade da criação de uma cultura coletiva para a análise de indicadores e promoção da melhoria contínua da assistência no setor hospitalar brasileiro.

A Etapa 2 possibilitou compreender que a melhoria assistencial é visada por meio do desenvolvimento de recursos humanos, do conhecimento e da pesquisa e da gestão participativa, observar que evidências são consideradas limitadas em relação ao contexto onde a organização é inserida, perceber que há lacunas na sistematização das experiências e barreiras no fluxo de informações e considerar que o uso de indicadores para dimensionar a demanda pode ser eficaz no contexto da saúde pública. Gestores dessa etapa utilizam indicadores como fonte de gerenciamento de recursos escassos. Os principais indicadores utilizados pelos entrevistados dessa etapa são nomeados como assistências, de qualidade, de recursos humanos e financeiros. Destacam-se os indicadores assistenciais e de recursos humanos para estabelecimento de prioridades de atendimento e a sua aplicação estratégica, incluindo recursos financeiros no contexto do modelo participativo de gestão.

$\mathrm{Na}$ Etapa 3, foi possível compreender como o modelo TQM orienta o planejamento estratégico da organização, verificar o uso de indicadores como meio para atingir a sustentabilidade fi- nanceira, econômica e assistencial do hospital, comprovar o uso de evidências e a renovação do estoque de conhecimentos como fator crucial para a gestão eficiente conforme o modelo corporativo, entender a necessidade de sistematizar as evidências e as experiências de profissionais e equipes para aquisição da meIhoria da qualidade assistencial. Gestores dessa etapa utilizam indicadores como fonte de informações para a sustentabilidade econômica e assistencial. Duas categorias de indicadores foram nomeadas como fundamentais para o dimensionamento estratégico: indicadores assistenciais e indicadores financeiros. Suas utilidades estratégicas principais são dimensionar o atendimento, melhorar a qualidade da assistência, atingir custos mínimos e planejar a sustentabilidade econômica.

Quanto às contribuições teórico-empíricas, conclui-se que o compartilhamento de informações é essencial à aplicação do sistema de indicadores investigado, o uso de indicadores na organização hospitalar pública privilegia o desenvolvimento do conhecimento e o uso de indicadores na organização filantrópica integra elementos da AD e da GCE com ênfase na gestão da qualidade como fator estratégico.

O uso compartilhado de um sistema de indicadores por um grupo de hospitais de diversas naturezas necessita de maior integração para atingir a competição baseada em valor quanto à meIhoria do desempenho setorial. Por outro lado, diferentes configurações organizacionais determinam a priorização de indicadores distintos para atingir prioridades estratégicas. No hospital público, os indicadores são priorizados para gestão estratégica da demanda. No hospital privado, as informações originadas por indicadores de desempenho são utilizadas para melhorar a qualidade da assistência e gerir custos com visão estratégica planejada.

Os resultados limitam-se ao uso gerencial de sistemas de indicadores hospitalares, hospitais gerais públicos e filantrópicos. É necessário comprová-los em contextos semelhantes para verificar a possibilidade de generalização. 0 estudo pretendeu atingir o nível estratégico da gestão hospitalar, podendo ser detalhado em pesquisas futuras nos níveis tático e operacional. A metodologia é uma contribuição do estudo à pesquisa em administração no ambiente hospitalar, tradicionalmente marcado pelo modelo clínico de investigação.

\section{Nota de agradecimento}

Os autores agradecem o apoio do comitê gestor do SIPAGEH, das direções e dos colaboradores dos hospitais participantes da pesquisa, ao Instituto de Pesquisa de Mercado (IPM-UNISINOS) e ao Ministério Público do Trabalho da $4^{\underline{a}}$ Região. 


\section{REFERÊNCIAS}

Ahire, S. L, Golhar, D. I, \& Waller, M. A. (1996). Development and validation of TQM implementation constructs. Decision Science, 27(1), 23-56.

Bardin, L. (2011). Análise de conteúdo. São Paulo: Edições 70.

Barney, J. (1991). Firm resources and sustained competitive advantage. Journal of Management, 17(1), 99-120.

Báscolo, E, Yavich, N, \& León, A. S. de. (2006). El proceso de interacción investigadores y tomadores de decisiones: un estudio de caso. Cadernos de Saúde Pública, 22, S47-S56.

Bittar, O. J. N. (2004). Indicadores de qualidade e quantidade em saúde. Revista de Administração em Saúde, 6 (22), 15-18.

Carman, J. M., Shortell, S. M., Foster, R. W., Hughes, E. F., Boerstler, H., O’Brien, J. L., \& O'Connor, E. J. (2010). Keys for successful implementation of total quality management in hospitals. Health Care Management Review, 35(4), 283-293.

Cherchiglia, M. L. \& Dallari, S. G. (2006). Tempo de mudanças: sobrevivência de um hospital público. RAE eletrônica, 5(2). Recuperado em 12 novembro, 2013, de http://www.scielo.br/scielo.php?script=sci_pdf\&pi $d=S 167656482006000200007 \& n g=e n \& n r m=i s o \& t l n g=p t$.

Corrao, S., Arcoraci, V., Arnone, S., Calvo, L., Scaglione, R., Bernardo, C. Di, Legalla, R., Caputi, A. P. \& Licata, G. (2009). Evidence-based knowledge management: an approach to effectively promote good healthcare decision-making in the information era. Internal and Emergency Medicine, 4(2), 99-106.

Cunha, J. A. C, da, \& Corrêa, H. L. (2013). Avaliação de desempenho organizacional: um estudo aplicado em hospitais filantrópicos. $R A E-R e$ vista de Administração de Empresas, 53(5), 485-499.

Denzin, N. K. \& Lincoln, Y. S. (2007). Planejamento da pesquisa qualitativa: teorias e abordagens (2a ed.). São Paulo: Artmed.

Donabedian, A. (1981). Criteria, norms and standards of quality: what do they mean? American Journal of Public Health, 71(4), 409-412.

Donabedian, A. (2005). Evaluating the quality of medical care. Milbank Quarterly, 83(4), 691-729.

Escrivão, A, Jr. (2007). Uso da informação na gestão de hospitais públicos. Ciência e Saúde Coletiva, 12(3), 655-666.

Flick, U. (2014). An introduction to qualitative research (5th ed.). London: Sage.

Forgia, G. M, La, \& Couttolenc, B. F. (2009). Desempenho hospitalar no Brasil: em busca da excelência. São Paulo: Singular.

Gonçalo, C. R, \& Borges, M. L. (2010). Organizações de saúde intensivas em conhecimento: um estudo no contexto de serviços de alta complexidade. Saúde e Sociedade, 19(2), 449-461.

Grant, R. M. (1996). Toward a knowledge-based theory of the firm. Strategic Management Journal, 17(2), 109-122.

Gurgel, G. D, Jr, \& Vieira, M. M. F. (2002). Qualidade total e administração hospitalar: explorando disjunções conceituais. Ciência e Saúde Coletiva, 7(2), 325-334.

Hronec, S. M, \& Andersen, A. (1994). Sinais vitais: usando medidas de desempenho da qualidade, tempo e custo para traçar a rota para o futuro da empresa. São Paulo: Makron Books.

Institute of Medicine. (2001). Crossing the quality chasm: a new health system for the 21 century. Washington, DC: National Academy Press.
Juran, J, \& Gryna, F. (1991). Controle da qualidade - Handbook: conceitos, políticas e filosofia da qualidade. São Paulo: Makron Books; São Paulo: McGraw Hill.

Khattab, S, Al, \& Aborumman, A. H. (2011). Healthcare service quality: comparing public and private hospitals in Jordan. International Business Management, 5(5), 247-254.

Kovner, A. R, Pfeffer, J, \& Fine, D. (2010). What more evidence do you need? Harvard Business Review, 8(5), 123-127.

Lei n. 8.080, de 19 de setembro de 1990. (1990). Lei Orgânica da Saúde. Dispõe sobre as condições para a promoção, proteção e recuperação da saúde, a organização e o funcionamento dos serviços correspondentes e dá outras providências.

Miguel, P. A. C. (Coord). (2012). Metodologia de pesquisa em engenharia de produção e gestão de operações (2a ed.). Rio de Janeiro: Elsevier; Rio de Janeiro: Abepro.

Miles, I., Kastrinos, N., Flanagan, K., Bilderbeek, R., Hertog, P. Den, Huntink, W., \& Bouman, M. (1995). Knowledge-intensive business services: users, carriers and sources of innovation. EIMS publication, (15).

Ministério da Saúde. (2004). Reforma do sistema de atenção hospitalar brasileiro. Brasília: Ministério da Saúde.

Nonaka, I, \& Krogh, G, Von. (2009). Perspective- tacit knowledge and knowledge conversion: controversy and advancement in organizational knowledge creation theory. Organization Science, 20(3), 635-652.

Nonaka, I, \& Takeuchi, H. (1997). Criação de conhecimento na empresa: como as empresas japonesas geram a dinâmica da inovação (14a ed.). Rio de Janeiro: Campus.

Paladini, E. P. (2011). Avaliação estratégica da qualidade (за ed. rev.). São Paulo: Atlas.

Pfeffer, J, \& Sutton, R. I. (2006). Evidence-based management. Harvard Business Review, 84(8), 63-74.

Porter, M, \& Teisberg, E. O. (2005). Redefining health care: creating value-based competition on results. Massachusetts: Harvard Business School Press.

Prahinsky, C, \& Benton, W. C. (2004). Supplier evaluations: communication strategies to improve supplier performance. Journal of Operations Management, 22(1), 39-62.

Reay, T, Berta, W, \& Kohn, M. K. (2009). What's evidence in evidence-based management? Academical Management Perspective, 23(4), 5-18.

Richardson, R. J. (2007). Pesquisa social: métodos e técnicas (за ed. rev. ampl.). São Paulo: Atlas.

Robin, M. (2008). Revisiting a TQM research project: the quality improvement activities of TQM. Total Quality Management, 19(8), 751-761.

Rousseau, D. M, Manning, J, \& Denyer, D. (2008). Evidence in management and organization science: assembling the field's full weight of scientific knowledge through synthesis. Academy of Management, 2(1), 475-515.

Rummler, G. A, \& Brache, A. P. (1992), Melhores desempenhos das empresas: ferramentas para a melhoria da qualidade e da competitividade. São Paulo: Makron Books.

Schout, D, \& Novaes, H. M. (2007). Do registro ao indicador: gestão da produção da informação assistencial nos hospitais. Ciência e Saúde Coletiva, 12(4), 935-944.

Silva, K. S. B, Bezerra, A. F. B, Souza, I. M. C, \& Gonçalves, R. F. (2010). 
Conhecimento e uso do sistema de informações sobre orçamentos públicos em saúde (SIOPS) pelos gestores municipais, Pernambuco, Brasil. Cadernos de Saúde Pública, 26(2), 373-382.

Sistema de Indicadores Padronizados para a Gestão Hospitalar. (2008). Recuperado em 17 dezembro, 2008, de http://www.projeto.unisinos. $\mathrm{br} / \mathrm{sipageh} /$

Slack, N, Chambers, S, Harrison, A, \& Jhonston, R. (1997). Administração da produção. São Paulo: Atlas.

Strambach, S. (2008). Knowledge-intensive business services (KIBS) as drivers of multilevel knowledge dynamics. Services, Technology and Management, 10(2), 152-174.

Sveiby, K. E. (2008). KM in a noruegian hospital. Recuperado em 21 abril, 2010, de http://www.sveiby.com/articles/KMCaseHospital.pdf
White, K. R, Lemak, C. H, \& Griffith, J. R. (2011). Improving healthcare management education using principles from Baldrige and evidence-based management. Journal of Health Administration Education, 28(3), 187-207.

Wurster, C. J, Lichtenstein, B. B, \& Hogeboom, T. (2009). Strategic, political, and cultural aspects of IT implementation: improving the efficacy of an IT system in a large hospital. Journal of Health Management, 54(3), 191-206.

Yin, R. K. (2001). Estudo de caso: planejamento e métodos (2a ed.). Porto Alegre: Bookman.

Zanella, L. C. H. (2009). Metodologia de estudo e de pesquisa em administração. Florianópolis: Departamento de Ciências da Administração/UFSC. 